\title{
Fluxgate offset study
}

\author{
P. Ripka, M. Pribil, M. Butta
}

Czech Technical University, 16627 Prague, Czech Republic

\begin{abstract}
Offset and its long-term stability is a weak point of fluxgate sensors. Even the ultrastable sensors kept at no vibrations and stable temperature at magnetic observatories show offset drift. Such drift of fluxgate tri-axial sensor can be only partly corrected by scalar resonance magnetometer. Periodical calibration of absolute reading should be made using non-magnetic theodolite. In this paper we study the origin of fluxgate offset. We distinguish the real magnetic sensor offset from the offset contributions originating in false $2^{\text {nd }}$ harmonics signal which leaks to the sensor output from the distortion in the excitation signal, or which is borne as harmonic distortion when the signal processing electronics is subjected to large $1^{\text {st }}$ harmonic signal leaking from the excitation. We analyze the offset dependence on the angular position of the sensor core and its response to large field shocks. The experiments give an indication that only a part of the magnetic offset stems from a remanence of magnetically hard core regions. The residual part may be caused by magnetostrictive signal, belonging to false signal contributions, but not considered in previous studies.
\end{abstract}

Index Terms—Fluxgate, Magnetic field sensor, Magnetometer.

\section{INTRODUCTION}

$T^{\mathrm{H}}$ HE performance of the fluxgate sensors is often limited by

the offset and its stability with temperature and time. The origin of the offset is, however, not much discussed in the recent literature after the classical works of Gordon [1] and Weiner [2]. Offset stability is also closely related to the sensor noise. A systematic study of offset on sensors based on new core materials is therefore needed.

Offset changes after large field shocks (perming) are reduced with increasing excitation amplitude. With the miniaturization of the fluxgates their operating frequency is usually increased in order to reach sufficient sensitivity. While the optimum excitation frequency of classical $2 \mathrm{~cm}$ diameter sensor is 10 to $20 \mathrm{kHz}$, for $10 \mathrm{~mm}$ ring this may be $100 \mathrm{kHz}$ and for integrated fluxgate with $1 \mathrm{~mm}$ core the optimum frequency may reach $1 \mathrm{MHz}$. With the increasing excitation frequency and decreasing dimensions, the parasitic capacitive couplings start to play an important role.

In this paper we show how to separate the sensor magnetic offset, i.e. the component of the offset due to imperfection of the magnetic core, from the offset caused by parasitic effects. The method is simple: it is based on commutation of the sensor excitation coil ("exc" commutator) and sensing coil ("sens" commutator) as schematically shown in Fig. 1. This approach is extended from the technique suggested by Mario Acuna and described in [3]. Contrary to the approach used in [3] we perform offset measurements always in the open loop, so that we need not consider the offset of the feedback amplifier. This brings the necessity of calibration of the sensor sensitivity before each measurement.

We also study the properties of the magnetic offset after shocks of large external field.

Manuscript received March 7, 2014. Corresponding author: P. Ripka (email: ripka@fel.cvut.cz).

Digital Object Identifier inserted by IEEE

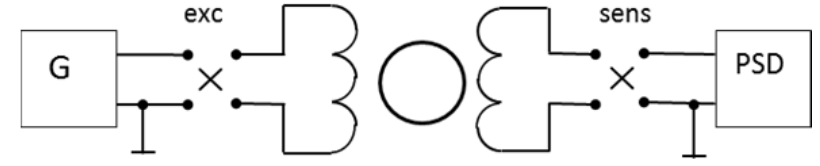

Fig. 1 Schematics of the fluxgate sensor excited by the generator $G$ and at the output connected to lock-in amplifier (phase-sensitive detector PSD.

\section{SOURCES OF FLUXGATE OFFSET}

The possible sources of the measured offset are:

1. Magnetic offset $B_{0 \mathrm{mag}}$

It is equivalent to the sensor response to the same magnetic field. The output therefore changes polarity when commutating the sensing winding. As the sensor is based on $2^{\text {nd }}$ harmonic output, the polarity of the magnetic offset is not changed with commutating the excitation: changing phase of excitation by $180^{\circ}$ changes the phase of $2^{\text {nd }}$ harmonic output signal by $360^{\circ}$.

\section{Inductive offset $B_{0 \text { ind }}$}

The $2^{\text {nd }}$ harmonic distortion in the excitation current and the leakage of this signal to the sensor output through inductive coupling. It changes sign with either commutation.

\section{Capacitive offset $B_{0 \text { cap }}$}

The $2^{\text {nd }}$ harmonic distortion in the excitation current and leakage to the sensor output through capacitive coupling. It changes sign with either commutation, however the parasitic capacitances $\mathrm{C}_{11}, \mathrm{C}_{12}, \mathrm{C}_{21}, \mathrm{C}_{22}$ are not necessarily equal. This causes asymmetry in the calculated offset levels.

\section{Distortion offset $B_{0 \text { dist }}$}

The $2^{\text {nd }}$ harmonic distortion in the detector. It does not change sign with any commutation. Amplitude of this offset depends on the amplitude of the fundamental frequency signal at the output of the sensor. This should be ideally zero, but in reality this signal depends on the asymmetry of the sensor, which may change in time. 


\section{Lock-in amplifier offset $\mathrm{B}_{0 \text { off }}$}

DC offset of the lock-in amplifier does not change sign with any commutation. DC offset can be easily verified by switching the excitation off and we assume that it is corrected before the measurement.

From the previous analysis we can derive rules for the measured offset values shown in Table I:

TABLE I

OFFSET CONTRIBUTIONS TO THE SENSOR OUTPUT DEPENDS ON THE POLARITY OF THE EXCITATION AND SENSING COILS

\begin{tabular}{ccc}
\hline \hline Exc & Sens & output \\
\hline+ & + & $\mathrm{B}_{++}=\mathrm{B}_{0 \mathrm{mag}}+\mathrm{B}_{0 \text { ind }}+\mathrm{B}_{0 \mathrm{C} 1}+\mathrm{B}_{0 \text { dist }}$ \\
+ & - & $\mathrm{B}_{+-}=-\mathrm{B}_{0 \mathrm{mag}}-\mathrm{B}_{0 \text { ind }}-\mathrm{B}_{0 \mathrm{C} 2}+\mathrm{B}_{0 \text { dist }}$ \\
- & - & $\mathrm{B}_{--}=-\mathrm{B}_{0 \mathrm{mag}}+\mathrm{B}_{0 \text { ind }}+\mathrm{B}_{0 \mathrm{C} 3}+\mathrm{B}_{0 \text { dist }}$ \\
- & + & $\mathrm{B}_{-+}=\mathrm{B}_{0 \text { mag }}-\mathrm{B}_{0 \text { ind }}-\mathrm{B}_{0 \mathrm{C} 4}+\mathrm{B}_{0 \text { dist }}$ \\
\hline
\end{tabular}

Unfortunately, in the system of equations summarized in Tab. 1 there are more unknown parameters than equations. However, the measured values allow checking the relative importance of the parasitic effects. Deeper analysis of the parasitic capacitances can be made using the measurements of the feedthrough signal at the fundamental frequency, where the capacitance feedthrough effect is half than that at 2nd harmonics. We found that for frequencies up to $100 \mathrm{kHz}$ the capacitive effects play minor role compared to the inductive ones. Capacitive feedthrough is dominant at integrated fluxgate, whose coils have lower number of turns and typical operation frequencies of $1 \mathrm{MHz}$.

\section{THE MEASURED SENSORS}

In this paper we show the values measured on two types of ring-core fluxgate sensors:

1. electrodeposited $28 \mathrm{~mm}$ diameter Permalloy ring

2. $10 \mathrm{~mm}$ diameter core etched from $30 \mu \mathrm{m}$ thick amorphous Vitrovac 6025.

The sensor parameters are shown in the Table II. Fig. 2 shows the hysteresis loop of the electrodeposited core with $\mathrm{H}_{\mathrm{c}}$ $=100 \mathrm{~A} / \mathrm{m}$. The advantage of this core is that due to low thickness of $6 \mu \mathrm{m}$ the eddy current losses are low. This is not the case of $30 \mu \mathrm{m}$ thick amorphous alloy: the coercivity at 100 $\mathrm{kHz}$ is 10 -times the DC coercivity (Fig. 3).

We used Agilent 33220A waveform generator to produce the sensor excitation current. Any DC component of the excitation current was blocked by serial $1 \mu \mathrm{F}$ capacitor as the DC current together with the sensor asymmetry would produce unpredictable offset. SR844 lock-in amplifier was used for the extraction of second harmonic from the induced voltage. The working point of each sensor was optimized for the best performance (low noise and low offset). The electrodeposited and amorphous sensors were always tuned at the output by parallel capacitors. For some of the measurements we also tuned the excitation current to increase its p-p level. The measured $2^{\text {nd }}$ harmonic distortion of the excitation current was only around $20 \mathrm{ppm}$, which is instrumental for reaching low inductive offset $\mathrm{B}_{0 \text { ind. }}$

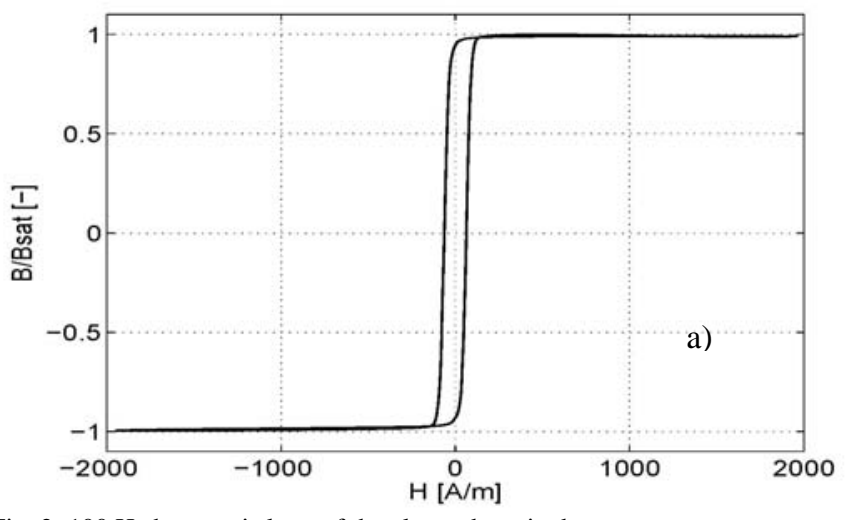

Fig. 2: $100 \mathrm{~Hz}$ hysteresis loop of the electrodeposited core

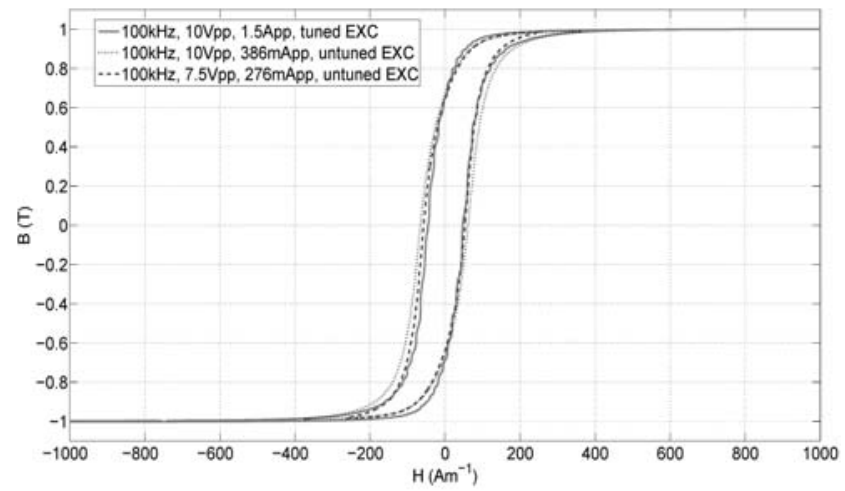

Fig. 3: Hysteresis loop of the amorphous core measured at the fluxgate working conditions

TABLE II

PARAMETERS OF THE MEASURED SENSORS

\begin{tabular}{|c|c|c|c|}
\hline Parameter & unit & sensor 1 & sensor 2 \\
\hline $\begin{array}{l}\text { Core } \\
\text { - material } \\
\text { - thickness } \\
\text { - outer diameter } \\
\text { - inner diameter }\end{array}$ & $\begin{array}{l}\mu \mathrm{m} \\
\mathrm{mm} \\
\mathrm{mm}\end{array}$ & $\begin{array}{l}\text { crystalline } \\
\text { electrodep. } \\
\text { Fe81Ni19 } \\
6 \\
28 \\
18\end{array}$ & $\begin{array}{l}\text { amorphous } \\
\text { etched } \\
\text { Vitrovac } 6025 \\
30 \\
10 \\
8\end{array}$ \\
\hline $\begin{array}{l}\text { Excitation windin } \\
\text { - number of turns } \\
\text { - wire diameter }\end{array}$ & $\begin{array}{l}\mathrm{T} \\
\mathrm{mm}\end{array}$ & $\begin{array}{l}270 \\
0.18\end{array}$ & $\begin{array}{l}65 \\
0.3\end{array}$ \\
\hline $\begin{array}{l}\text { Sensing coil } \\
\text { - length } \\
\text { - number of turns } \\
\text { - wire diameter }\end{array}$ & $\begin{array}{l}\mathrm{mm} \\
\mathrm{T} \\
\mathrm{mm}\end{array}$ & $\begin{array}{l}18 \\
200 \\
0.18\end{array}$ & $\begin{array}{l}8 \\
185 \\
0.1\end{array}$ \\
\hline $\begin{array}{l}\text { Excitation } \\
\text { - frequency }\end{array}$ & $\mathrm{kHz}$ & 25 & 100 \\
\hline - amplitude & V p-p & 4 & $10 \mathrm{~V}$ \\
\hline Sensing & & & \\
\hline - tuning capacitor & $\mathrm{nF}$ & - & 6.85 \\
\hline - sensitivity & $\mu \mathrm{V} / \mathrm{T}$ & 6 & 80 \\
\hline
\end{tabular}

The process of finding the optimum working point is described only for the first sensor. In general the process is complex as the sensor is a highly non-linear system, which usually includes two resonant circuits: one in the excitation 
and the second one at the sensor output. The adjustable parameters are excitation frequency, amplitude and waveform shape, tuning capacitance in the excitation and tuning capacitance at the sensor output. The sensor design is usually optimized for maximum sensitivity and minimum noise; in this paper we focus on minimum offset.

\section{MEASUREMENTS ON THE SENSOR WITH AMORPHOUS CORE}

The shape of the tuning curve strongly depends on frequency. Fig. 4 shows sensitivity as a function of the excitation current (p-p value). At each point the tuning capacitor at the sensor output was adjusted for maximum sensitivity, i.e. on the top of the resonance curve. Thus, what we see in Fig. 2 are not resonant curves but changes of their amplitude with increasing excitation current. At $\mathrm{f}_{\text {exc }}=50 \mathrm{kHz}$ the increase is monotonous, which is a natural trend: by increasing the current amplitude we also increase the speed of change of magnetic field which is responsible for the amplitude of the output voltage. At $\mathrm{f}_{\mathrm{exc}}=50 \mathrm{kHz}$ we observe new effect: The quality factor of the non-linear parametric resonance circuit is increased for certain current levels by parametric amplification. At $\mathrm{f}_{\mathrm{exc}}=75 \mathrm{kHz}$ we already observe unstable behaviour: for current levels between $150 \mathrm{~mA}$ and $250 \mathrm{~mA}$ p-p, the circuit becomes a parametric oscillator.

Such oscillation can be killed by decreasing the quality factor e.g. by adding resistor in series with the output coil. It is always questionable how the high sensitivity in the vicinity of unstable region can degrade offset stability and sensor noise.

Fig. 5 shows noise figures for $100 \mathrm{kHz}$ excitation: while the noise for untuned $\mathrm{I}_{\mathrm{exc}}=7.5 \mathrm{~V} \mathrm{p}-\mathrm{p} / 276 \mathrm{~mA} \mathrm{p}-\mathrm{p}$ is 306pT/ $\sqrt{\mathrm{Hz}} @ 1 \mathrm{~Hz}$, it drops down to 87 pT pT/ $\sqrt{ } \mathrm{Hz} @ 1 \mathrm{~Hz}$ when the excitation amplitude is increased to untuned 10 V/386 mA p-p. This noise level at this frequency is not changed after excitation tuning, which for the same excitation voltage level increases the current to $1.5 \mathrm{~A}$ p-p. Also the sensitivity is almost the same for tuned $(80 \mathrm{~V} / \mu \mathrm{T})$ and untuned $(174 \mathrm{~V} / \mu \mathrm{T})$ excitation circuit. However the increase of the white noise and also ultra-low-frequency noise for untuned excitation is observed.

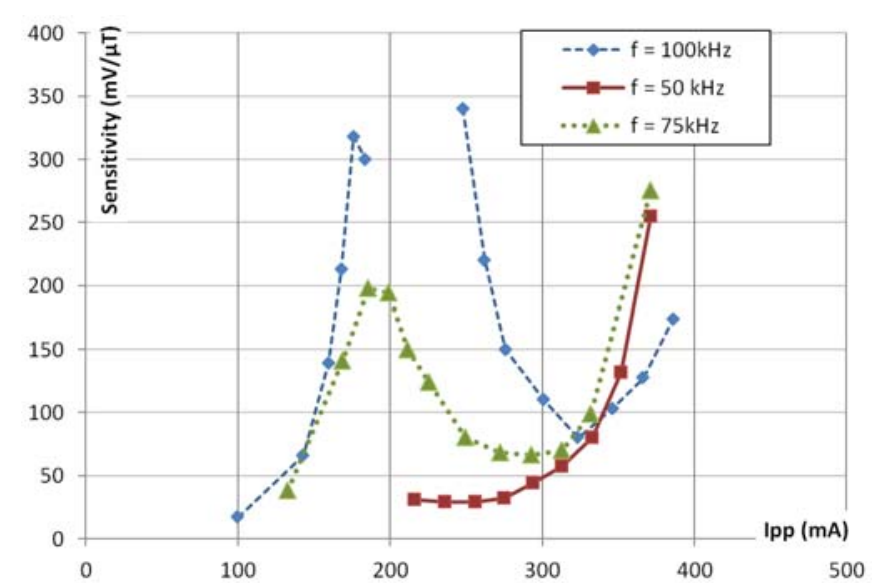

Fig. 4 Sensitivity of amorphous sensor as a function of excitation current for 50,75 and $100 \mathrm{kHz}$
Fig. 6 shows offset of this sensor after switch-on: after the warming period the offset variations are within $1.5 \mathrm{nT}$ band.

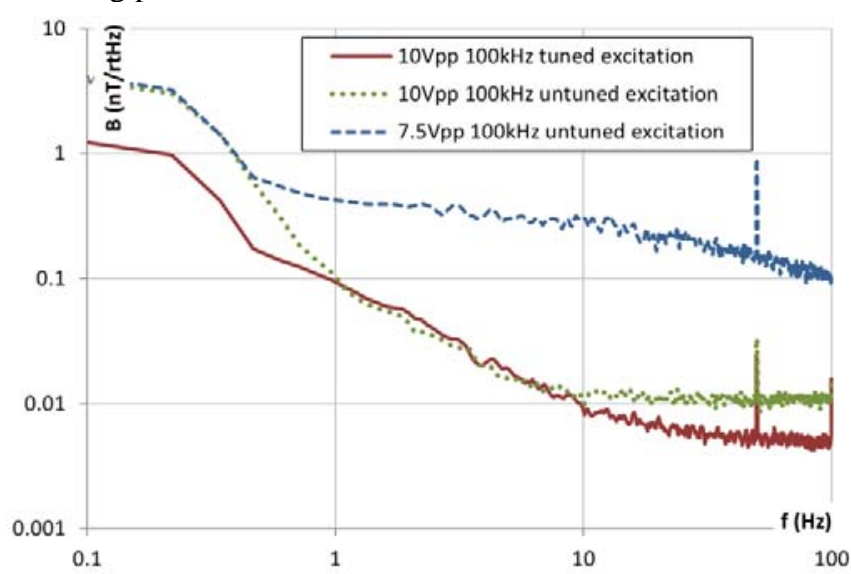

Fig. 5 Noise for amorphous sensor excited at $100 \mathrm{kHz}$

7.5 V p-p corresponds to $276 \mathrm{~mA}$ p-p, 10 V/untuned to $386 \mathrm{~mA} \mathrm{p}-\mathrm{p}$ and 10 V/tuned to 1.5 A p-p

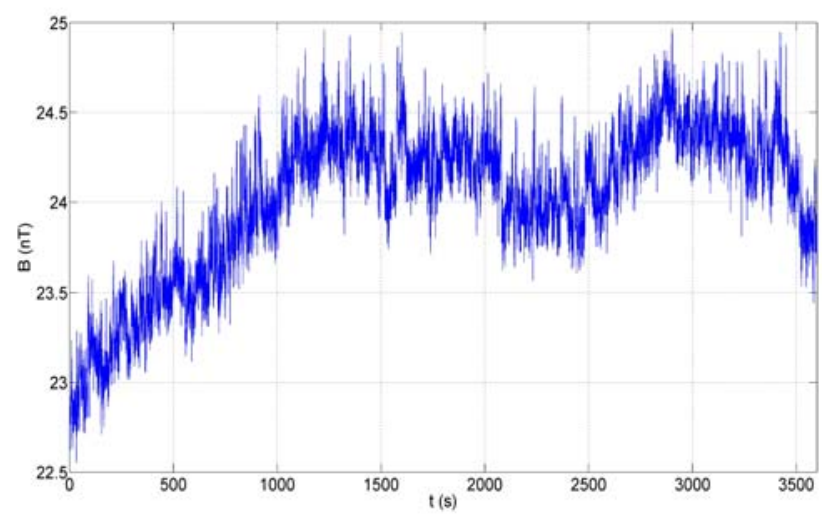

Fig. 6 Offset drift of amorphous sensor 1h after switch-on.

The offset level measured at optimized working conditions is shown in Table III together with the minimum offset values measured for the electrodeposited sensor.

The amorphous sensor has very high magnetic offset. It should be noted that this sensor has arbitrary angular position of its core with respect to the pickup coil and the mechanical construction does not allow a core rotation. There is a common believe that the fluxgate offset is caused by magnetically hard regions in the sensor core which have larger remanence caused by local defects, inclusions and other imperfections. If this were true, these regions can be remagnetized by an external magnetic field of sufficient intensity. We therefore applied subsequent $1 \mathrm{~T}$ field shocks of alternating polarity and measured the offset level between them. We used magnetic 4-layer and 6-layer cylindrical shieldings for the offset testing. The residual field inside the shielding is tested by turning the sensor by $180^{\circ}$. The $1 \mathrm{~T}$ shock field is generated by electromagnet. The offset response to these shocks was reproducible and the individual offset components are shown in Tab. 3. 
TABLE III

OFFSET COMPONENTS AFTER 1T FIELD SHOCKS

\begin{tabular}{lccc}
\hline \hline & $\mathrm{B}_{0}^{+}$ & $\mathrm{B}_{0}{ }^{-}$ & $\begin{array}{c}\text { residual offset } \\
\mathrm{B}_{0 \mathrm{R}}=\left(\mathrm{B}_{0}^{+}+\mathrm{B}_{0}^{-}\right) / 2\end{array}$ \\
\hline \hline & & & \\
\hline $\mathrm{B}_{0 \text { mag }}(\mathrm{nT})$ & 5.6 & -11.9 & -3.1 \\
$\mathrm{~B}_{\text {0ind }}(\mathrm{nT})$ & -2.4 & -2.4 & -2.4 \\
$\mathrm{~B}_{\text {0dist }}(\mathrm{nT})$ & -2.4 & 3.4 & 0.5 \\
\hline
\end{tabular}

The possible sources of the residual magnetic offset of 3.1 nT may be some magnetically very hard regions which can create false 2nd harmonic signal with amplitude independent of magnetic history of the core. Another source can be false $2^{\text {nd }}$ harmonic signal originating from magnetostriction effects.

\section{Measurements On the SEnSOR With ELECTRODEPOSITED CORE}

The main advantage of Sensor 2 is the possibility to rotate the sensor core with respect to the pick-up coil. This allows finding minimum offset or minimum feedthrough signal. However, measurement of the offset components at different rotational position gives important information about the sensor quality. A good example is shown in Fig. 7. Sensor 1 has small noise and it can be adjusted for zero offset. However, the offset was strongly dependent on the angular position of the core (Fig. 7a). The switching exercise according to Table 1 has shown that the magnetic offset $B_{\text {mag }}$ is very small, but the "inductive offset" $\mathrm{B}_{0 \text { ind }}$ is extraordinary. The "inductive offset" $\mathrm{B}_{0 \text { ind }}$ requires some 2nd harmonic, which is always present in the excitation circuit, and some non-symmetry which causes coupling between the excitation and sensing coils. This can be caused by non-uniformity of the excitation winding and by non-homogeneity of the core crosssectional area (in our case caused by the variation of the core thickness) and by non-homogeneity of its magnetic properties. Such sensor should not be used for critical applications such as in space magnetometers, as the vibrations during the lift-off may cause core movement and resulting large shift in offset.

A typical offset dependence for a "good sensor" of the same type is shown in Fig. 7b: here the magnetic offset contribution dominates.

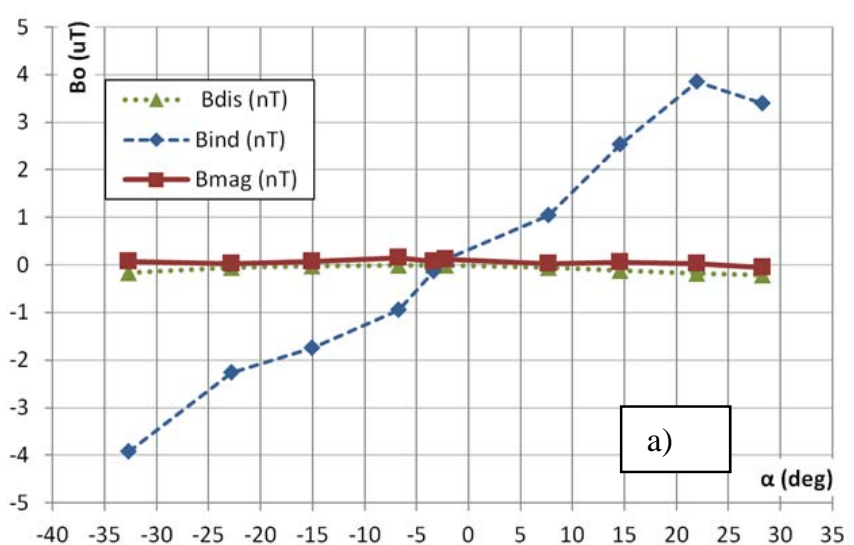

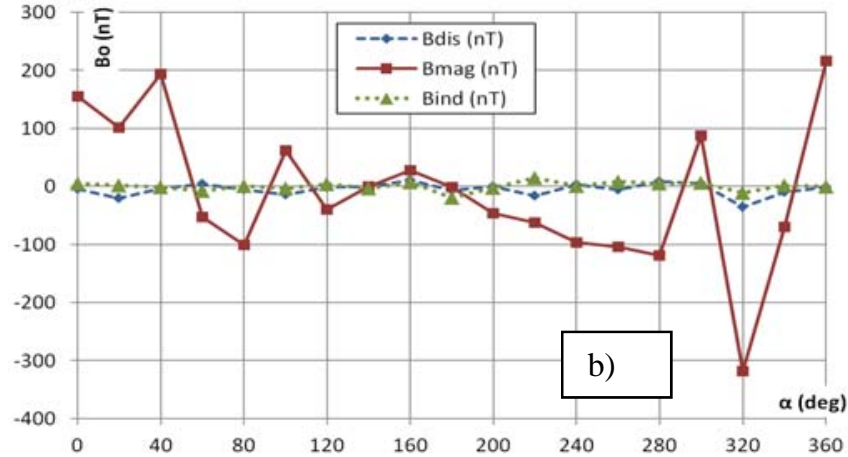

Fig. 7. Offset components vs. core rotation angle for the electrodeposited sensor a) sensor 1 with poor homogeneity b) sensor 2 with standard homogeneity

Fig. 8 shows that the offset stability of the electrodeposited sensor is 10-times lower than that of amorphous sensor.

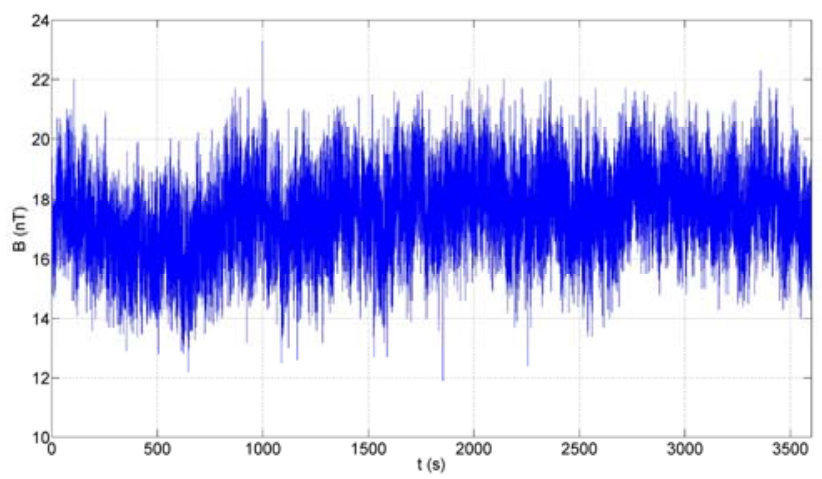

Fig. 8. Offset stability of electrodeposited sensor.

\section{CONCLUSIONS}

By flipping of fluxgate input and output coils we are able to make decomposition of the main offset components: magnetic and false contributions. From the reaction of the offset to large field shocks we found, that only a part of the magnetic offset is caused by core remanence; the possible explanation for residual magnetic offset is the false $2^{\text {nd }}$ harmonics from magnetostriction effects. The offset level depends on the angular position of the sensor core with respect to the pick-up coil and on the magnetic history of the sensor.

\section{ACKNOWLEDGMENT}

This work has been supported by the Grant Agency of the Czech Republic (GACR) under the grant P102/12/2177.

\section{REFERENCES}

[1] Gordon, D.; Lundsten, R.; Scarzello, J., "Offset and noise in fluxgate magnetometers," Magnetics, IEEE Transactions on, vol.6, no.4, pp.818,818, Dec 1970

[2] Weiner, M., "Magnetostrictive offset and noise in flux gate magnetometers," Magnetics, IEEE Transactions on, vol.5, no.2, pp.98,105, Jun 1969

[3] 0. V. Nielsen, J. R. Petersen, A. Fernandez, B. Hernando, P. Spisak, F. Primdahl and N. Moser: Analysis of a fluxgate magnetometer based on metallic glass sensors, Meas Sci. Technol. 2 (1991) 435440

[4] P. Ripka, W.G. Hurley: Excitation efficiency of fluxgate sensors, Sensors and Actuators A 129 (2006), pp.75-79 